\title{
Early Rehabilitation Programs Improve Pain and Disability in Patients with Lumbar Disc Protrusion: A Prospective Con- trolled Study
}

\author{
Emilian Tarcău 1,4, Dorina Ianc 1, 4 , Elena Sîrbu 2, *, Doriana Ciobanu 1,4, Ioan Cosmin Boca ${ }^{1,4}$ and Florin Marcu ${ }^{3}$ \\ 1 Department of Physical Education, Sport and Physiotherapy, University of Oradea, Romania; emi- \\ lian.tarcau@yahoo.com (E.T.); dorina.ianc@yahoo.com (D.I.); doriana.ciobanu@yahoo.com (D.C.); ic- \\ boca@yahoo.com (I.C.B.) \\ 2 Department of Physical Therapy and Special Motricity, Faculty of Physical Education and Sport, West Uni- \\ versity of Timisoara, Romania; elena.sirbu@e-uvt.ro (S.E.) \\ 3 Department of Psychoneuroscience and Rehabilitation, University of Oradea, Romania; \\ mfmihai27@yahoo.com \\ 4 Human Performance Research Center, Department of Physical Education, Sport and Physiotherapy, Uni- \\ versity of Oradea, Romania \\ * Correspondence: elena.sirbu@e-uvt.ro (S.E.)
}

\begin{abstract}
Low back pain is a common problem in the active population, and the second reason for visiting a physician. In patients with lumbar disc protrusion, the nucleus pulposus bulges against the disc and the latter protrudes into the spinal column, but the annulus fibrosus remains intact. The purpose of this study was to prove that starting an early complex rehabilitation treatment results into pain and disability reduction, and increased muscle strength and mobility in patients with lumbar disc protrusions. We performed a prospective cohort study, enrolling 60 patients (25 men and 35 women) aged between 26 to 76 years, diagnosed with lumbar disc protrusion. Patients in the experimental group registered significant improvements in all studied variables (pain, mobility, muscle strength, disability) after 6 months of treatment. The results of our study suggest that, in the lumbar disc disease, a combined rehabilitation program may be more effective in terms of pain and disability reduction, if it starts early after diagnosis. The current study proves the importance of combining electrotherapy with hydrotherapy and physical therapy. Patients who received this treatment combination showed an extremely significant improvement in pain relief, and reduction of functional disability after 6 months of treatment.
\end{abstract}

Keywords: low back pain; physical therapy; disability; hydrotherapy

\section{Introduction}

Low back pain is a common problem in the active population, and the second reason for visiting a physician. Besides the fact that low back pain causes a substantial morbidity, as it is the main reason for disabilities in the active population, it also generates major expenses consisting of treatment costs, lost working days and decreased productivity [1].

In patients with lumbar disc protrusion, the nucleus pulposus bulges against the disc and the latter protrudes into the spinal column, but the annulus fibrosus remains intact.

The monthly prevalence of lumbar disc disease is estimated at $43 \%$ of the population; thus, low back pain is the second reason for visiting a GP or consultant [2]. In Romania, the prevalence of low back pain in adults is high (62\%), second only to headaches $(79 \%)$ in the ranking of painful disorders [3].

The high prevalence of the lumbar disc degenerative disease is explained by the presence of several risk factors, which are more and more present in our society: weight lifting, ageing, sedentary lifestyle, smoking, obesity and genetics [4]. 
Lifting a weight with while flexing the trunk and extending the upper limbs will change the spinal alignment and centre of balance (body's centre of gravity) at the abdomen level. As a result, the spine must bear both the weight of the upper body, and the weight of the load to be lifted or lowered. Repetitive physical activities that overload the lumbar spine in flexion can eventually cause the protrusion and destruction of the posterior annulus fibrosus.

Several studies reported that ageing is an important risk factor for disc bulging and/or protrusion $[5,6]$.

It is well known that smoking causes vasoconstriction, and nicotine decreases blood flow to the vertebral bodies. In turn, this decrease can reduce the flow of nutrients to the disc and predispose to disc degeneration [7].

There is a linear dependence relationship between the volume of physical activity and state of health, so that the most physically active people present the lowest risk of developing the disease. Thus, the lack of sports activities is a significant risk factor for the development and progression of lumbar disc degeneration [8].

On the other hand, obese people are more likely to develop lumbar disc degeneration and protrusion [9].

Recent studies have revealed that genetic factors may also be involved in lumbar disc disease and in other inflammatory rheumatic diseases [10,11]. The identification of two collagen IX alleles was associated with the lumbar disc disease. Moreover, disc degeneration has been shown to be related to an aggrecan gene polymorphism, a vitamin D receptor and matrix metalloproteinase-3 gene alleles [12].

The purpose of this study was to prove that starting an early complex rehabilitation treatment results into pain and disability reduction, and increased muscle strength and mobility in patients with lumbar disc protrusions.

\section{Materials and Methods}

\subsection{Study Design and Participants}

We performed a prospective cohort study, enrolling 60 patients ( 25 men and 35 women) aged between 26 to 76 years, diagnosed with lumbar disc protrusion.

Patients were recruited at the Ceres Hotel Treatment Centre from Băile 1 Mai, Romania, from July 2021 to January 2022, and the study was performed over a period of 6 months.

This study complied with the principles outlined in the Helsinki Declaration and was approved by the local Ethics Committee (approval no. 1947/14.07.2021). All participants provided their written informed consent before being included in the study.

The inclusion criteria were: $25-80$ years of age, low back pain for more than three months, an MRI confirmed diagnosis of lumbar disc protrusion (without dural compression), ability to perform a physical therapy program. The exclusion criteria were: indication for acute surgery, previous surgery on the same lumbar spinal level, sciatica, presence of severe spinal pathology (spinal tumour, spinal fracture, spinal stenosis or radiculopathy, fibromyalgia, inflammatory and infectious spinal diseases). Chronic pain relief drug users, refusal to participate in the research, neoplasms of any sort, severe comorbidities, mental illness, are also all exclusionary factors.

\subsection{Assessments}

The participants' demographic characteristics such as age, gender, weight, height and body mass index (BMI) were recorded. In addition, the presence of radiculopathies and the elapsed period (in months) from diagnosis to the start of treatment were noted.

According to one's personal reasons (availability, adherence, health costs etc), the patients were assigned either to a control $(n=30)$ or experimental group $(n=30)$. The control group ( $\mathrm{Gr}$ A) received only a classical electrotherapy program. In addition, the 
patients in the experimental group (Gr B) received a complex individualized physical exercise therapy program associated with hydrotherapy and electrotherapy.

All assessments were made before intervention and 6 months later by the same physiotherapist, who was blinded to the treatment groups.

Spinal mobility was assessed using the lateral lumbar flexion (LLF) testing, index-ground (IG), lumbar Schober tests for flexion (LS), and Inverted Schober test for extension (ILS) [13-17].

In the case of muscle strength evaluation, both the strength of the flexor and extensor muscles of the trunk was tested. To assess the abdominal muscle strength, the number of trunk flexions the subject could perform in a 30 seconds interval was recorded. In the case of evaluating the strength of the lumbar extensor muscles, the number of correct executions of spine extensions in a 30-second interval was noted.

The Short Form McGill Pain Questionnaire (SF-MPQ) was used to determine the pain level [18-20]. The SF-MPQ is a multidimensional measure of perceived pain in adults with chronic pain, including pain due to lumbar disc diseases [21]. The questionnaire contains a total of 15 descriptors ( 4 affective and 11 sensory) which are rated on an intensity scale: " 0 " = none, " 1 "= mild, " 2 "= moderate, " 3 "= severe. The existing data confirm the reliability, validity, and responsiveness of SF-MPQ in patients with chronic pain [22].

Additionally, the degree of pain was evaluated using a visual analogue scale (VAS) on a 10-cm scale, with 0 indicating "no pain" and 10 indicating "worst pain".

The Oswestry Disability Questionnaire (ODQ) was used to measure the limitation in everyday life activities [23]. There is evidence supporting its validity and reproducibility [24]. The ODQ is based on 10 sections with six levels each, assessing the limitation of various activities of daily living. The values range from 0 (the best health state) to 100 (the worst health state). For each section of the questionnaire, the total possible score is 5 . The first statement was scored 0 , and consecutive statements were scored from 1 to 5 . The total score was then divided by the total possible score and expressed as a percentage to produce the Oswestry Disability Index (ODI). The ODI is interpreted as follows: $0-20 \%$, minimal disability; $21-40 \%$, moderate disability; $41-60 \%$, severe disability; $61-80 \%$, crippled; $81-100 \%$, patients are either bed-bound or exaggerate their symptoms.

\subsection{Interventions and Procedures}

The patients in the experimental group ( $\mathrm{Gr} B$ ) received a 10-day inpatient rehabilitation program, once a day. Moreover, they continued the daily physical therapy exercise program after hospital discharge.

All patients in Gr B attended a complex rehabilitation program consisting of: hydrotherapy, physical therapy and electrotherapy.

Patients were treated with $37^{\circ} \mathrm{C}$ water therapy (hydrotherapy) in a therapeutic pool for $30 \mathrm{~min}$ a day, 5-days a week for a total duration of 10 days. In "Băile 1 Mai", the thermal water used for the treatment has a mineralization that varies around $1 \mathrm{~g} / \mathrm{l}$ with mainly negative ions (anions) - bicarbonates, sulphates and positive ions (cations) - calcium, sodium and magnesium.

A physical therapy program was given to all patients for $45 \mathrm{~min}$ a day, 5-days a week for a total duration of 10 days. The exercises consisted of active resisted movement of trunk flexion and extension, pelvic tilt, abdominal and trunk muscles strengthening, and trunk mobility exercises. The dose and intensity of the exercises were set and progressed over time. The program was individualized according to the patients' specific impairments.

After completing the exercise program, electrotherapy was applied to each patient. The electrotherapy procedures consisted of: 1) Transcutaneous electrical nerve stimulation (TENS) in conventional mode, symmetric biphasic, $90 \mathrm{~Hz}$, for $15 \mathrm{~min}$; 2) Interferential current (IFC) in quadripolar mode, at $100 \mathrm{~Hz}$ frequency, for $10 \mathrm{~min}$; 3) Magnetic field therapy in continuous form (sedative effect), for $15 \mathrm{~min}$. 
TENS and IFC were performed using the Chattanooga Intelect Neo combined device. The magnetic field was delivered through a Physiomed MAG-Expert device with a field strength of 1-100 Gauss (adjustable in steps of one Gauss) and a frequency range from 1 to $100 \mathrm{~Hz}$, with two completely independent channels and a treatment timer.

After completing the 10 days program of complex physical therapy in the hotel's treatment centre, all patients received a written outline and description of the exercise program and they were reminded to carry out the exercises regularly.

The patients in the control group were only treated with electrotherapy, for 10 days, in a hospital. The electrotherapy protocol was the same as for the experimental group.

\subsection{Data Analysis}

For data analysis, we used the SPSS 15.0 statistical analysis program. For the quantitative analysis of the numerical variables we used the mean and standard deviation, and for the categorical variables we used the percentage and mean. We analyzed the normality of data distribution using the Kolmogorov-Smirnov test. For the intergroup analysis of both the initial and the final results, we used the independent samples T-test, as we have a normal data distribution (the Kolmogorov-Smirnov test, $\mathrm{p} \geq 0.05$ ).

\section{Results}

The distribution of the patients' parameters in each group was homogeneous (differences were not statistically significant) according to the studied variables: age, gender, body mass index, time elapsed from diagnosis and start of the treatment, presence of radiculopathies (table 1, the Kolmogorov-Smirnov test, $\mathrm{p} \geq 0.05$ ).

Table 1. Subjects' characteristics (mean \pm SD / \%), time from diagnosis to first treatment (mean \pm SD) and presence of radiculopathy (\%)

\begin{tabular}{|c|c|c|}
\hline Characteristics & Group A & Group B \\
\hline Age (years) & $53.30 \pm 11.20$ & $52.97 \pm 11.04$ \\
\hline BMI $\left(\mathrm{kg} / \mathrm{m}^{2}\right)$ & $29.28 \pm 4.71$ & $29.89 \pm 4.41$ \\
\hline Time elapsed (months) & $9.0 \pm 6.73$ & $10.63 \pm 5.32$ \\
\hline \multirow[t]{2}{*}{ Gender (\%) } & 46.7 & 36.7 \\
\hline & 53.3 & 63.3 \\
\hline \multirow[t]{3}{*}{ Radiculopathy (\%) } & 26.7 & 33.3 \\
\hline & 30.0 & 33.3 \\
\hline & 43.3 & 33.3 \\
\hline
\end{tabular}

There was no significant difference between the groups in terms of pain, muscle strength, mobility and disability at the initial evaluation (table 2).

Table 2. Comparisons of the initial parameters between the two groups (confidence interval 95) (mean \pm SD)

\begin{tabular}{llll}
\hline Initial parameters & Group A & Group B & $\mathbf{p}$ \\
\hline SF-MPQ & $12.57 \pm 6.027$ & $12.77 \pm 4.897$ & 0.888 \\
\hline FTF & $-17.02 \pm 10.841$ & $-17.57 \pm 4.321$ & 0.797 \\
\hline LS & $13.82 \pm 0.932$ & $13.92 \pm 1.325$ & 0.736 \\
\hline ILS & $8.93 \pm 0.452$ & $8.05 \pm 0.702$ & 0.058 \\
\hline Right LLF & $12.90 \pm 3.573$ & $13.81 \pm 3.008$ & 0.289 \\
\hline Left LLF & $12.62 \pm 3.314$ & $13.85 \pm 2.948$ & 0.134 \\
\hline
\end{tabular}




\begin{tabular}{llll}
\hline LF strength & $10.00 \pm 5.206$ & $12.63 \pm 2.109$ & 0.013 \\
\hline LE strength & $9.80 \pm 6.641$ & $13.00 \pm 2.101$ & 0.015 \\
\hline ODQ & $17.37 \pm 5.605$ & $17.53 \pm 4.455$ & 0.899 \\
\hline ODQ (\%) & $34.73 \pm 11.209$ & $35.07 \pm 8.909$ & 0.899 \\
\hline
\end{tabular}

SF-MPQ = Short Form McGill Pain Questionnaire; FTF = fingertip-to-floor distance; LS = Lumbar Schober test;

ILS = Inverted lumbar Schober test; LLF = lateral lumbar flexion; LF strength = strength for flexor muscles; LE strength = strength for extensor muscles; ODQ = Oswestry Disability Questionnaire; * $p<0.05$

The final intergroup comparison (between groups)demonstrates that there are significant differences regarding pain intensity $\mathrm{t}(48.95)=6.98, \mathrm{p}<0,05$; fingertip-to-floor distance $\mathrm{t}(43.87)=2.886, \mathrm{p}<0,05$; Schober test $\mathrm{t}(56.42)=-5.146, \mathrm{p}<0,05$; ODQ score $\mathrm{t}(57.87)=$ 3.627, $\mathrm{p}<0,05$; the disability index (QDI) $\mathrm{t}(57.12)=-3.007, \mathrm{p}<0,05$. At 6 months, the experimental group had improved more than the control group in regards to pain (SF-MPQ), disability (ODQ, ODI) and LS scores $(\mathrm{P}<0.05)$. There was no statistically significant difference in lateral left flexion $\mathrm{t}(57.53)=0.569, \mathrm{p}>0,05$; lateral right flexion $\mathrm{t}(57.69)=0.227$, $\mathrm{p}>0,05$; trunk flexion strength $\mathrm{t}(40.43)=2-110, \mathrm{p}>0,05$; trunk extension strength $\mathrm{t}(36.75)=$ $0.801, \mathrm{p}>0,05$ between the two groups (Table 3 ).

Table 3. Comparisons of the final parameters between the two groups (mean \pm SD)

\begin{tabular}{lccl}
\hline Final parameters & Group A & Group B & P \\
\hline SF-MPQ & $2.70 \pm 2.120$ & $7.47 \pm 3.137$ & $0.000^{*}$ \\
\hline FTF & $-11.20 \pm 1.126$ & $-18.12 \pm 6.102$ & $0.005^{*}$ \\
\hline LS & $11.20 \pm 1.126$ & $12.48 \pm 1.334$ & $0.000^{*}$ \\
\hline ILS & $8.38 \pm .537$ & $8.04 \pm .666$ & 0.034 \\
\hline Right LLF & $14.13 \pm 3.386$ & $13.65 \pm 3.094$ & 0.571 \\
\hline Left LLF & $13.48 \pm 2.845$ & $13.67 \pm 3.061$ & 0.821 \\
\hline LF strength & $14.80 \pm 5.517$ & $12.47 \pm 2.501$ & 0.093 \\
\hline LE strength & $14.30 \pm 7.489$ & $13.13 \pm 2.763$ & 1.167 \\
\hline ODQ & $8.13 \pm 3.785$ & $15.60 \pm 3.616$ & $0.001^{*}$ \\
\hline ODI $(\%)$ & $17.93 \pm 6.269$ & $23.13 \pm 7.099$ & $0.004^{*}$ \\
\hline
\end{tabular}

SF-MPQ = Short Form McGill Pain Questionnaire; FTF = fingertip-to-floor distance; LS = Lumbar Schober test;

ILS = Inverted lumbar Schober test; LLF = lateral lumbar flexion; LF strength = strength for flexor muscles; LE strength = strength for extensor muscles; ODQ = Oswestry Disability Questionnaire; * $p<0.05$

The intra group comparison (within the control group) proves there are significant differences between the pretest-posttest values for pain intensity $t(29)=13.91, p<0,05$; fingertip-to-floor test $\mathrm{t}(29)=1.41, \mathrm{p}<0,05$; Schober test $\mathrm{t}(29)=2.81, \mathrm{p}<0,05$; disability $\mathrm{t}(29)=$ $17.00, \mathrm{p}<0,05$ (table 4 ). The percentage score obtained in the evaluation of the quality of life decreased on average by 11.93 points (95\% CI [10.52, 13.34]).

Table 4. The results and statistical comparisons of the pre-treatment and 6 month evaluations in the control group (mean $\pm \mathrm{SD})(95 \% \mathrm{CI})$

\begin{tabular}{lrrl}
\hline Parameters & Pre-treatment & After 6 months & \multicolumn{1}{l}{$\mathbf{P}$} \\
\hline SF-MPQ & $12.77 \pm 4.897$ & $7.47 \pm 3.137$ & $0.000^{*}$ \\
\hline FTF & $-17.57 \pm 4.321$ & $-18.12 \pm 6.102$ & $0.001^{*}$ \\
\hline
\end{tabular}




\begin{tabular}{lccl}
\hline LS & $13.92 \pm 1.325$ & $13.66 \pm 1.132$ & $0.005^{*}$ \\
\hline ILS & $8.05 \pm 0.702$ & $8.04 \pm 0.666$ & 0.841 \\
\hline Left LLF & $13.81 \pm 3.008$ & $13.65 \pm 3.094$ & 0.249 \\
\hline Right LLF & $13.85 \pm 2.948$ & $13.67 \pm 3.061$ & 0.284 \\
\hline LF strength & $12.63 \pm 2.109$ & $12.47 \pm 2.501$ & 0.538 \\
\hline LE strength & $13.00 \pm 2.101$ & $14.13 \pm 2.763$ & 0.670 \\
\hline ODQ & $17.53 \pm 4.455$ & $11.60 \pm 3.616$ & $0.000^{*}$ \\
\hline ODI (\%) & $35.07 \pm 8.909$ & $23.13 \pm 7.099$ & $0.000^{*}$ \\
\hline
\end{tabular}

SF-MPQ = Short Form McGill Pain Questionnaire; FTF = fingertip-to-floor distance; LS = Lumbar Schober test;

ILS = Inverted lumbar Schober test; LLF = lateral lumbar flexion; LF strength = strength for flexor muscles; LE strength = strength for extensor muscles; ODQ = Oswestry Disability Questionnaire; * $p<0.05$

Patients in the experimental group registered significant improvements in all studied variables (pain, mobility, muscle strength, disability) after 6 months of treatment (table 5). This means that post-intervention, a statistically significant difference was found in the P values of SF-MPQ, FTF, LS, ILS, LLF, LE, ODQ.

Table 5. The results and statistical comparisons of the pre-treatment and 6 month evaluations in the experimental group (mean $\pm \mathrm{SD})(95 \% \mathrm{CI})$

\begin{tabular}{llll}
\hline Parameters & Pre-treatment & After 6 months & p \\
\hline SF-MPQ & $12.57 \pm 6.027$ & $2.73 \pm 1.982$ & $0.000^{*}$ \\
\hline FTF & $-17.02 \pm 10.841$ & $-11.20 \pm 11.617$ & $0.000^{*}$ \\
\hline LS & $13.82 \pm 0.932$ & $14.40 \pm .884$ & $0.005^{*}$ \\
\hline ILS & $8.93 \pm 0.452$ & $8.38 \pm 0.537$ & $0.004^{*}$ \\
\hline Left LLF & $12.90 \pm 3.573$ & $14.13 \pm 3.386$ & $0.001^{*}$ \\
\hline Right LLF & $12.62 \pm 3.314$ & $13.84 \pm 2.845$ & $0.005^{*}$ \\
\hline LF strength & $10.00 \pm 5.206$ & $14.80 \pm 5.517$ & $0.001^{*}$ \\
\hline LE strength & $9.80 \pm 6.641$ & $14.30 \pm 7.489$ & $0.000^{*}$ \\
\hline ODQ & $17.37 \pm 5.605$ & $8.13 \pm 3.785$ & $0.000^{*}$ \\
\hline ODI $(\%)$ & $34.73 \pm 11.209$ & $17.93 \pm 6.269$ & $0.000^{*}$ \\
\hline
\end{tabular}

SF-MPQ = Short Form McGill Pain Questionnaire; FTF = fingertip-to-floor distance; LS = Lumbar Schober test;

ILS = Inverted lumbar Schober test; LLF = lateral lumbar flexion; LF strength = strength for flexor muscles; LE strength = strength for extensor muscles; $\mathrm{ODQ}=$ Oswestry Disability Questionnaire; * $p<0.05$

The analysis of the correlation between the time elapsed from diagnosis to the start of the rehabilitation treatment program and the level of pain, as well as disability index, was performed using the Pearson correlation coefficient.

In the experimental group there is a significant positive correlation between the time elapsed from diagnosis to treatment administration and level of pain $\mathrm{r}(28)=0.81, \mathrm{p}=.000$ (fig. 1 - left. The sooner treatment is started, the lower the pain at the end of the treatment period. In the control group it is noticed that there is no significant correlation between the time elapsed from diagnosis to the start of treatment and the level of pain $r(28)=0.17$, $\mathrm{p}=.930$ (fig. 1 - right). 

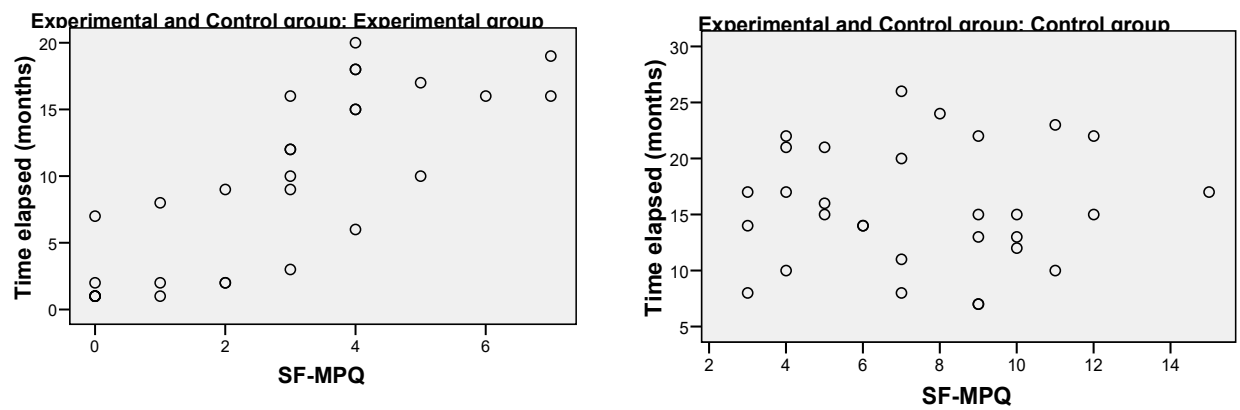

Figure 1. Correlation between the time elapsed from diagnosis and the level of pain after 6 months for the experimental group (left) and control group (right).

In the experimental group, we noticed a significant positive association between the elapsed period from diagnosis to the start of treatment and the disability index $\mathrm{r}(28)=$ $0.62, p=.000$ (fig. 2- left). The sooner treatment is started, the more reduced is the discomfort caused by the disease in 6 months of treatment. In the control group, we noticed a significant negative correlation between the time elapsed from diagnosis to treatment administration and the disability index $r(28)=-0.58, p=.001$ (fig. 2- right). The longer the period elapsed from diagnosis to treatment, the greater the discomfort created by the disease.
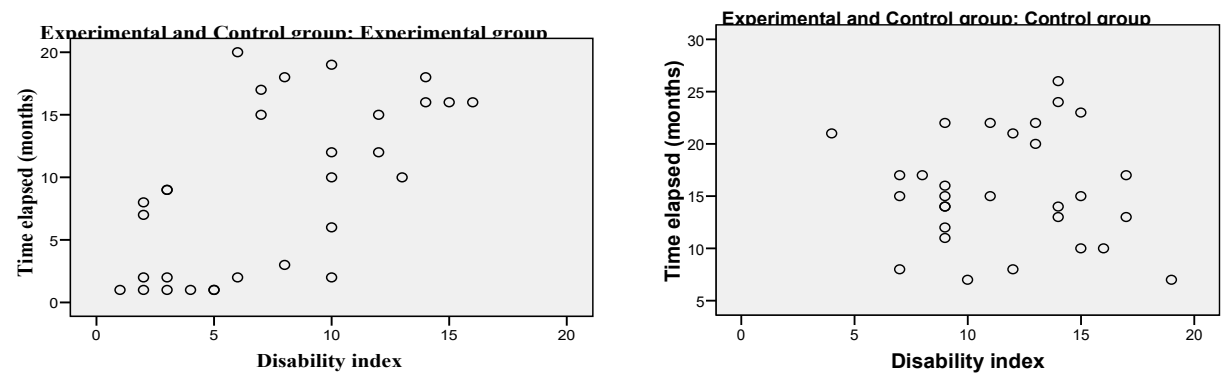

Figure 2. Correlation between the time elapsed from diagnosis and the disability index after 6 months for the experimental group (left) and control group (right).

\section{Discussion}

According to the Global Burden of Disease 2017 Study, the global prevalence and years lived with disability of back pain has increased between 1990 and 2017, registering the maximum prevalence in the 45-49 age group in 2017 [26].

In fact, the first degenerative changes in the disc appear after the age of 30 . At the level of the annulus fibrosus there is a decrease in elasticity, while at the level of the nucleus pulposus its hydrophilic capacity decreases, favouring the occurrence of traumatic lesions with the partial or total rupture of the annulus fibrosus, which will cause disc protrusion or disc herniation on the midline or lateral.

Patients with lumbar disc protrusion may experience back pain, reduced mobility, weakness in the legs and feet, disability during the person's daily functioning and decreased quality of life.

According to specialized studies, the conservative treatment has proven to be successful in the treatment of patients with lumbar disc protrusion, leading to pain relief, improved mobility and symptoms severity. Over time, various methods of rehabilitation treatment in patients with lumbar disc protrusion have been studied, including patient education, behavioral therapies, back school, exercises, physical therapy, balneotherapy and electrotherapy (ultrasound, TENS, interferential currents, laser) [27- 30].

Moreover, surgical treatment has not been shown to be more effective than conservative treatment in reducing the severity of symptoms or improving the quality of life 
in patients with lumbar disc disease in the midterm or long term follow-up as referred by Gugliotta M. et al. Although pain was relieved more quickly in patients who received surgical treatment (persistent at the 3-week follow-up), the difference was no longer present after 3 months [31].

The results of our study show that subjects in both groups registered better outcomes after a 6 months rehabilitation program. However, after comparing the final parameters between the two groups, the experimental group who underwent electrotherapy, hydrotherapy and physical therapy had significantly improved in regards to pain (SF-MPQ), disability (ODQ, ODI) and LS scores.

Unlike the control group, the comparison of the initial and final results for the experimental group shows that there are significant differences between the pretest and posttest in all parameters. Thus, patients from the experimental group registered significant improvements in pain, mobility, muscle strength and disability after 6 months of combined treatment.

The benefits of electrotherapy modalities, delivered alone or in combination with other interventions, in relieving pain, improving range of motion or disability, were reported in several studies [28, 30,32, 33, 34]. On the other hand, hydrotherapy has proven beneficial effects in pain reduction and lumbar mobility in patients with low back pain $[29,35]$.

Although there has been a lot of debate around the effectiveness of various interventions (alone or in combination), there are few studies in the literature that combine electrotherapy with physical therapy and hydrotherapy in treating patients with low back pain [28-35].

Another finding of our study was that patients included in the experimental group showed a significant positive correlation between the time elapsed from diagnosis to treatment and pain intensity. The sooner the treatment starts, the lower the pain at the end of the treatment period.

Moreover, we noticed a significant positive association between the period elapsed from diagnosis to the start of treatment and disability in these patients. So, we can state that the sooner the treatment starts, the more the discomfort created by the disease decreases after 6 months of complex treatment. In return, no positive significant correlation was observed in the control group.

The results of our study suggest that, in the lumbar disc disease, a combined rehabilitation program may be more effective in terms of pain and disability reduction, if it starts early after diagnosis. Similar results were reported by other authors who demonstrated that early intervention displayed significant improvement in pain, disability and socioeconomic outcomes (such as return-to-work and healthcare utilization) [36 -40]

However, other authors think that the effect of early physical therapy on pain and disability are significant, but the improvements were modest and did not last long [36, 37].

Our findings may stimulate further researches with longer follow-up periods and larger patient groups.

\section{Conclusions}

The current study proves the importance of combining electrotherapy with hydrotherapy and physical therapy. Patients who received this treatment combination showed an extremely significant improvement in pain relief, and reduction of functional disability after 6 months of treatment. The earlier the rehabilitation treatment is implemented, the lower the pain intensity and level of disability associated with the lumbar disease.

Author Contributions: Conceptualization, E.T.; methodology, E.S. and F.M.; software, D.C.; validation, D.I.; formal analysis, D.I.; investigation, E.T. and F.M.; resources, I.C.B.; data curation, F.M.; writing-original draft preparation, D.I. and E.S.; writing-review and editing, D.C. and I.C.B.; visualization, D.C.; supervision, E.S.; project administration, E.T. and I.C.B. All authors have read 
and agreed to the published version of the manuscript. All authors have equal contribution to this article.

Funding: This research received no external funding.

Institutional Review Board Statement: The study was conducted according to the guidelines of the Declaration of Helsinki, and approved by the Spa Treatment and Work Capacity Recovery Society - Baile 1 Mai branch - Hotel Ceres, (approval no. 1947/14.07.2021).

Informed Consent Statement: Informed consent was obtained from all subjects involved in the study.

Data Availability Statement: The datasets either used, analyzed, or both, during the current study are available from the corresponding authors on reasonable requests.

Acknowledgments:In this section, you can acknowledge any support given which is not covered by the author contribution or funding sections. This may include administrative and technical support, or donations in kind (e.g., materials used for experiments).

Conflicts of Interest: The authors declare no conflict of interest.

\section{References}

1. Dalstra, J.A.A.; Kunst, A.E.; Borrell, E. et al. Socioeconomic differences in the prevalence of common chronic disease: an overview of eight European countries. International Journal of Epidemiology. 2005,34,316-326.

2. Hoy, D.; Bain, C.; Williams, G.; March, L.; Brooks, P.; Blyth, F. et al. A systematic review of the global prevalence of low back pain. Arthritis Rheum. 2012;64(6):2028-37.

3. Busse, R.; Schreyogg, J.; Smith P.C. Variability in healthcare treatment costs amongst nine EU countries - results from the Health basket project, Health Econ. 2008,17,51-58.

4. Deyo, R.A.; Weinstein, J.N. Low back pain. New Engl J Med. 2001,344,363-370.

5. Matsumoto, M.; Okada, E.; Toyama, Y.; Fujiwara, H.; Momoshima, S.; Takahata, T. Tandem age-related lumbar and cervical intervertebral disc changes in asymptomatic subjects. Eur Spine J. 2013,22(4),708-13

6. Hung, I.Y.; Shih, T.T.; Chen, B.B.; Guo, Y.L. Prediction of Lumbar Disc Bulging and Protrusion by Anthropometric Factors and Disc Morphology. Int J Environ Res Public Health. 2021,18(5),2521.

7. Huang, W.; Qian, Y.; Zheng, K.; Yu, L.; Yu, X. Is smoking a risk factor for lumbar disc herniation. European Spine Journal. 2016,25,168-176.

8. Elfering, A.; Semmer, N.; Birkhofer, D.; Zanetti, M.; Hodler, J.; Boos, N. Young Investigator Award 2001 Winner: Risk Factors for Lumbar Disc Degeneration Individuals. Journal Spine. 2002,27(2),125-134.

9. Schroeder, D.G.; Guyre. C.A.; Vaccaro, A.R. The epidemiology and pathophysiology of lumbar disc herniation. Spine Surgery. 2016,28(1),2-7.

10. Sirbu, E.; Buleu, F.; Tudor, A.; Dragan, S. Vitamin D and disease activity in rheumatoid arthritis patients: a retrospective study in a Romanian cohort. Acta Biochimica Polonica. 2020,67(2), 267-272.

11. Stoicănescu, L.D.; Cevei, M.L.; Sirbu, E.M.; Zdrîncă, M.M.; Muțiu, G. Unusual occurrence of avascular necrosis with bilateral involvement and ankylosing spondylitis, meningioma and Hodgkin lymphoma. Rom J Morphol Embryol. 2019,60(3),1003-1007.

12. Ala-Kokko, L. Genetic risk factors for lumbar disc disease. Ann Med. 2002,34(1),42-7).

13. Radulović, N.; Pavlović, R.; Mihajlović, I.; Nikolić, S. Diagnostic of spinal column mobility using Schober's test for lumbal syndrome by application of physical therapy and sport recreation. European Journal of Physical Education and Sport Science. 2017,3,51-66.

14. Schober, P. The lumbar vertebral column in backache. Munchener Medizinish Wochenschrift.1937, 84, 336 - 338.

15. Moll, J.; Wright, V. Measurement of spinal movement. In: The lumbar spine and back pain, 3rd ed.;Jayson, M.I.V., Ed.;Edinburgh: Churchill Livingstone; 1987,11,215-234.

16. Sinaki, M.; Mokri, B. Low back pain and disorders of the lumbar spine. In: Physical medicine E rehabilitation; Braddom, R.L.; Buschbacher, R.M.; Dumitru, D.; Johnson, E.W.; Matthews, D.; Sinaki, M., Eds.; USA: WB Saunders Co.; 1996,39,813-850.

17. Tousignant, M.; Poulin, L.; Marchand, S.; Viau, A.; Place, C.The Modified-Modified Schober Test for range of motion assessment of lumbar flexion in patients with low back pain: A study of criterion validity, intra-and inter-rater reliability and minimum metrically detectable change. Disability and rehabilitation. 2005,27(10),553-9.

18. Strand, L.I.; Ljunggren, A.E.; Bogen, B.; Ask, T.; Johnsen, T.B. The Short-Form McGill Pain Questionnaire as an outcome measure: test-retest reliability and responsiveness to change. Eur J Pain. 2008,12(7),917-25. doi: 10.1016/j.ejpain.2007.12.013.

19. Webography W1. Available online: http://www.chcr.brown.edu/pcoc/shortmcgillquest.pdf (accessed on 30 November 2021).

20. Melzack, R. The short form McGill pain questionnaire. Pain, 1987,30,191-197.

21. Byrne, M.; Troy, A. et al. Cross-validation of the factor structure of the McGill Pain Questionnaire. Pain. 1982,13(2),193-201.

22. Dworkin, R.H.; Turk, D.C.; Trudeau, J.J.; Benson, C.; Biondi, D.M.; Katz, N.P.; Kim, M. Validation of the Short-form McGill Pain Questionnaire-2 (SF-MPQ-2) in acute low back pain. J Pain. 2015,16(4),357-66. doi: 10.1016/j.jpain.2015.01.012.

23. Fairbank, J.C.; Pynsent, P.B. The Oswestry disability index. Spine (Phila Pa 1976). 2000, 25(22), $2940-2952$. 
24. Tomkins-Lane, C.C.; Battié, M.C. Validity and reproducibility of self-report measures of walking capacity in lumbar spinal stenosis. Spine (Phila Pa 1976). 2010,35(23),2097-2102.

25. Catan, L.; Nemes, D.; Dragoi, M.; Poenaru, D.V.; Amaricai, E.; Popa, D.; Surducan, D.; Onofrei, R.; ... \& Cretu, O. Quality of life in patients with type III ankylosing spondylitis and secondary seronegative spondylarthropathies. Revista de Ortopedie si Traumatologie a Asociatiei de Ortopedie Româno-Italo-Spaniole. 2010,4,15.

26. Wu, A.; March, L.; Zheng, X.; Huang, J.; Wang, X.; Zhao, J.; Blyth, F.M.; Smith, E.; Buchbinder, R.; Hoy, D. Global low back pain prevalence and years lived with disability from 1990 to 2017: estimates from the Global Burden of Disease Study 2017. Ann Transl Med. 2020,8(6),299. doi: 10.21037/atm.2020.02.175.

27. Kolu, E.; Buyukavci, R.; Akturk, S.; Eren, F.; Ersoy, Y. Comparison of high-intensity laser therapy and combination of transcutaneous nerve stimulation and ultrasound treatment in patients with chronic lumbar radiculopathy: A randomized single-blind study. Pakistan journal of medical sciences.2018,34(3),530-534. https://doi.org/10.12669/pjms.343.14345

28. Facci, L.M.; Nowotny, J.P.; Tormem, F.; Trevisani, V.F. Effects of transcutaneous electrical nerve stimulation (TENS) and interferential currents (IFC) in patients with nonspecific chronic low back pain: randomized clinical trial. Sao Paulo Med J. 2011,129(4),206-16. doi: 10.1590/s1516-31802011000400003.

29. Mooventhan, A.; Nivethitha L. Scientific evidence-based effects of hydrotherapy on various systems of the body. N Am J Med Sci. 2014,6(5),199-209. doi:10.4103/1947-2714.132935

30. Zdrodowska, B.; Leszczyńska-Filus, M.; Leszczyński, R.; Błaszczyk, J. Comparison of the effect of laser and magnetic therapy for pain level and the range of motion of the spine of people with osteoarthritis lower back. Pol Merkur Lekarski. 2015 ,38(223),26-31.

31. Gugliotta, M.; da Costa, B.R.; Dabis, E.; Theiler, R.; Jüni, P.; Reichenbach, S.; Landolt, H., Hasler, P. Surgical versus conservative treatment for lumbar disc herniation: a prospective cohort study. BMJ open. 2016,6(12),e012938. https://doi.org/10.1136/bmjopen-2016-012938.

32. Ariel, E.; Levkovitz, Y.; Goor-Aryeh, I.; Ratmansky, M. The effects of TENS, interferential stimulation, and combined interferential stimulation and pulsed ultrasound on patients with disc herniation-induced radicular pain. J Back Musculoskelet Rehabil. 2021, doi: 10.3233/BMR-200302.

33. Ozkaraoglu, D.K.; Tarakci, D.; Algun, Z.C. Comparison of two different electrotherapy methods in low back pain treatment. J Back Musculoskelet Rehabil. 2020,33(2),193-199. doi: 10.3233/BMR-181199.

34. Yurdakul, O.V.; Beydoğan, E.; Yılmaz Yalçınkaya, E. Effects of physical therapy agents on pain, disability, quality of life, and lumbar paravertebral muscle stiffness via elastography in patients with chronic low back pain. Turk J Phys Med Rehabil. 2019,65(1),30-39. doi: 10.5606/tftrd.2019.2373.

35. Sawant, R.S.; Shinde, S.B. Effect of hydrotherapy based exercises for chronic nonspecific low back pain. Indian Journal of Physiotherapy \& Occupational Therapy. 2019,13(1),133-8.

36. Gatchel, R.J.; Polatin, P.B.; Noe, C.; Gardea, M.; Pulliam, C.; Thompson, J. Treatment- and cost-effectiveness of early intervention for acute low-back pain patients: a one-year prospective study. J Occup Rehabil. 2003,13(1),1-9. doi: 10.1023/a:1021823505774.

37. Molde Hagen, E.; Grasdal, A.; Eriksen, H.R. Does early intervention with a light mobilization program reduce long-term sick leave for low back pain: a 3-year follow-up study. Spine (Phila Pa 1976). 2003,28(20),2309-15. doi: 10.1097/01.BRS.0000085817.33211.

38. Fritz, J.M.; Magel, J.S.; McFadden, M. et al. Early physical therapy vs usual care in patients with recent-onset low back pain: A randomized clinical trial. JAMA. 2015,314(14), 1459-1467.

39. Childs, J.D.; Fritz, J.M.; Wu, S.S.; Flynn, T.W.; Wainner, R.S.; Robertson, E.K.; Kim, F.S.; George, S.Z. Implications of early and guideline adherent physical therapy for low back pain on utilization and costs. BMC health services research. 2015,15,150. https://doi.org/10.1186/s12913-015-0830-3.

40. Rhon, D.; Fritz, J. COMParative Early Treatment Effectiveness between physical therapy and usual care for low back pain (COMPETE): study protocol for a randomized controlled trial. Trials.2015,16,423. https://doi.org/10.1186/s13063-015-0959-8. 